\title{
Grooming online, sexting y problemas emocionales en adolescentes argentinos
}

\author{
Grooming online, sexting and emotional problems in Argentinean adolescents
}

Grooming online, sexting e problemas emocionais em adolescentes argentinos

\author{
Santiago Resett ${ }^{1}$ ORCID 0000-0001-7337-0617 \\ ${ }^{1}$ Universidad Católica Argentina
}

\begin{abstract}
Resumen: El grooming online hacia niños y adolescentes es una problemática de suma gravedad. El objetivo de esta investigación fue medir los niveles de grooming online padecido y si el género, la edad, el sexting y los problemas emocionales eran predictores de dicha conducta. Se constituyó una muestra intencional de 727 adolescentes entre 12 y 16 años (59\% mujeres) de dos escuelas privadas de la ciudad de Paraná, Argentina. Se detectó un 20\% de casos de grooming, con $17 \%$ recibiendo solicitaciones por parte de adultos y un $12 \%$ de interacciones con adultos. Más varones que mujeres sufrían de grooming -aunque marginalmente- e interacciones. También a mayor edad de los adolescentes se incrementaba linealmente el grooming, las solicitaciones e interacciones. Se observó que se predecía una varianza del $23 \%$ para el grooming, de $22 \%$ para solicitudes y $23 \%$ para interacciones, con la edad, la depresión y el sexting como los predictores significativos. Los resultados indicarían que esta problemática presenta porcentajes alarmantes en dicha población.
\end{abstract}

Palabras Clave: grooming, sexting, problemas emocionales, género, edad.

Abstract: Online grooming towards children and adolescents is a very serious problem. Thus, the objective of this research was to measure levels of online Grooming suffered and if gender, age, sexting and emotional problems (depression and anxiety) were predictors of such behavior. An intentional sample of 727 adolescents between 12 and 16 years old (59\% women) was constituted from two private schools in the city of Paraná, Argentina. $20 \%$ of grooming cases were detected - at least once in the last year -, with $17 \%$ receiving solicitations by adults and $12 \%$ interactions. More men than women suffered from grooming -albeit marginally- and interactions. Besides, at older teenagers, grooming, requests and interactions increased linearly. From hierarchical regressions it was observed that a variance of $23 \%$ was predicted for grooming, $22 \%$ for requests and $23 \%$ for interactions, with age, depression and sexting being significant predictors. These results would indicate that this problem presents alarming percentages in said population.

Key Words: grooming, sexting, emotional problems, gender, age. 
Resumo: O grooming online para crianças e adolescentes é um problema muito grave. O objetivo desta pesquisa foi medir os níveis de grooming online sofridos e se o gênero, a idade, o sexting e os problemas emocionais eram preditores de tal comportamento. Foi constituída uma amostra intencional de 727 adolescentes de 12 a 16 anos (59\% mulheres) de duas escolas particulares da cidade do Paraná, Argentina. Foram detectados $20 \%$ de casos de grooming, com $17 \%$ recebendo solicitações de adultos e $12 \%$ de interações com adultos. Mais homens do que mulheres sofreram grooming - embora marginalmente - e interações. Além disso, com o aumento da idade dos adolescentes se incrementaram linearmente o grooming, as solicitações e as interações. Observouse que o resultado previa uma variância de $23 \%$ para grooming, $22 \%$ para solicitações e $23 \%$ para interações, com a idade, a depressão e o sexting como preditores significativos. Os resultados indicam que este problema apresenta percentuais alarmantes na referida população.

Palavras-chave: grooming, sexting, problemas emocionais, gênero, idade.

Recibido: $15 / 11 / 2019$

Aceptado: 08/12/2020

\section{Cómo citar:}

Resett, S. (2021). Grooming online, sexting y problemas emocionales en adolescentes argentinos. Ciencias Psicológicas, 15(1), e-2397. doi: https://doi.org/10.22235/cp.v15i1.2397

Correspondencia: Santiago Resett, Universidad Católica Argentina, calle Laurencena 222 bis, Paraná, Entre Ríos, Argentina, CP 3100. Teléfono: 54 343-6101147.Email: santiago_resett@hotmail.com.

El grooming online se define como un proceso a través del cual un adulto con las tecnologías de la información y comunicación (TIC) trata de ganar la confianza de un niño o adolescente para crear o mantener un contacto sexual online o -posteriormente- de modo presencial (Kloess, Beech, \& Harkins, 2014; Smith, Thompson, \& Davidson, 2014; Wurtele \& Kenny, 2016). El proceso del grooming online hacia niños y adolescentes por parte de los adultos se compone de dos aspectos: la solicitación por parte de los adultos, por un lado, y la interacción sexual online con ellos, por el otro. El primero consiste en pedidos para que se involucren en actividades, conversaciones sexuales o para el intercambio de información sexual personal (Mitchell, Finkelhor, \& Wolak, 2007), mientras que el segundo aspecto consiste en interacciones de un adulto hacia el niño o adolescente (Gámez-Guadix, De Santisteban, \& Alcazar, 2017).

La diferencia de poder entre los niños o adolescentes y los adultos pueden volver a los adolescentes más vulnerables al contacto sexual con los adultos y a manejar dichos contactos de forma inadecuada, debido a su falta de maduración cognitiva y emocional (McRae et al., 2012; Wolak, Finkelhor, Mitchell, \& Ybarra, 2010). Por ejemplo, se detectó que la sintomatología depresiva adolescente se asociaba con un mayor riesgo de sufrir solicitaciones sexuales (Ybarra, Leaf, \& Diener-West, 2004). Si bien el grooming online puede ser perpetrado de un adolescente hacia otro (Ybarra \& Mitchell, 2008), es psicosocialmente más negativo cuando lo perpetra un adulto (Wolak et al., 2010). Por otra parte, las relaciones sexuales de los adolescentes con los adultos se asocian con importantes costos de salud física, como embarazo, enfermedades de transmisión sexual, entre otros (Manlove, Terry, Humen, \& Ikramullah, 2006; Wolak et al., 2010). También el sufrir grooming online se relaciona a una peor salud mental, como ansiedad, estrés, depresión, fobias, baja autoestima o suicidio (Montiel, Carbonell, \& Pereda, 2016). Debe ser tenido en cuenta que, por este motivo, la edad de consentimiento sexual en la Argentina -como en otros países de habla hispana, como España- es a partir de los 16 años, por lo cual si un adulto tiene contacto sexual con un menor de 16 años es un delito penal. En dicho país, el Código Penal en el 
artículo 131 lo incluyó en delitos contra la integridad sexual. No obstante, la edad de consentimiento sexual puede variar de nación a nación de acuerdo con la jurisprudencia legal.

Con respecto a los niveles del grooming online, la prevalencia de solicitación sexual en el último año fue de 5\%-15\% en adolescentes de 10 a 17 años en los Estados Unidos y Europa (Bergen et al., 2014). Otro estudio en los Estados Unidos arrojó cifras similares con un 9\% de adolescentes de la misma edad (Jones, Mitchell, \& Finkelhor, 2012). También un estudio en Holanda con adolescentes encontró que un $6 \%$ de los varones lo padecían, mientras que en las mujeres las cifras ascendían a un 19\% (Baumgartner, Valkenburg, \& Peter, 2010). De los pocos estudios existentes en idioma español, una investigación en España detectó que un 12\% de los adolescentes menores de 16 años informaban solicitaciones sexuales por parte de adultos, mientras que un 7\% señaló interacciones sexuales con ellos (Gámez-Guadix et al., 2017). Con respecto a las diferencias de género en esta conducta, las investigaciones señalan que las mujeres informan sufrirlo más frecuentemente que los varones, mientras que a mayor edad se lo padece en mayor medida (Mitchell, Wolak, \& Finkelhor, 2008; Wolak et al., 2010).

Otra conducta negativa online por parte de los adolescentes que en el último tiempo atrajo la atención de los investigadores y la comunidad es el sexting o sexteo en idioma español (Quesada, Fernández-González, \& Calvete, 2018). Este consiste en la creación y el intercambio de mensajes de texto, videos, imágenes o fotos con contenido sexual personal mediante la Internet, las redes sociales o el celular (Agustina \& Gómez-Durán, 2012; Barrense-Dias, Berchtold, Surís, \& Aire, 2018; Mitchell, Finkelhor, Jones, \& Wolak, 2012; Morelli, Bianchi, Baiocco, Pezzuti, \& Chirumbolo, 2016). El sexting es un factor de riesgo para el ajuste psicosocial de los sujetos, principalmente en los niños o adolescentes. Entre dichos comportamientos de riesgo asociados a esta variable, se pueden citar las conductas sexuales a edades tempranas (Benotsch, Snipes, Martin, \& Bull, 2012), una mayor probabilidad de sufrir de cyberbullying o agresión online (Crimmins \& Seigfried-Spellar, 2014; Quesada et al., 2018; Reyns, Burek, Henson, \& Fisher, 2013; Ricketts, Maloney, Marcum, \& Higgins, 2015). Además, otro de los grandes riesgos psicosociales para los adolescentes es que muchos de los que sufren grooming online, también pueden sufrir otras problemáticas relacionadas a las nuevas tecnologías, como el sexting o el cyberbullying (Wachs et al., 2017), aunque la interrelaciones entre dichas conductas prácticamente no ha sido investigada en el mundo (Machimbarrena et al., 2018). Una de las pocas investigaciones a este respecto detectó que quienes sufrían grooming online también tenían más probabilidad de sufrir sexting (Machimbarrena et al., 2018).

La solicitación e interacción sexual de los adultos hacia los adolescentes se ha vuelto una preocupación en los medios de comunicación, la comunidad educativa y académica. Sin embargo, no existen en la Argentina, como en otros países de la América Latina, investigaciones que hayan evaluado dicha conducta en adolescentes con instrumentos de sólidas propiedades psicométricas para evaluar sus niveles y si el género, la edad, los problemas emocionales y el sexting son predictores del mismo. De este modo, la importancia del presente trabajo es que se trata del primero en su tipo en examinar una problemática con vastas implicancias psicosociales, físicas y jurídicas, pero escasamente estudiada en la Argentina.

Los objetivos específicos del trabajo son: a) medir la incidencia del grooming online y su variación según el género y la edad en adolescentes de 12-16 años; b) determinar si los problemas emocionales (depresión y ansiedad), el sexting, el género y la edad son predictores del grooming.

\section{Método}

\section{Participantes}

Se constituyó una muestra intencional de 727 adolescentes de dos escuelas secundarias privadas de Paraná, Entre Ríos, Argentina, con edades de 12 años a 16, con $M_{\text {edad }}=15.01(D E=$ 
1.7); un 59\% eran mujeres y $41 \%$ varones. Un $38 \%$ cursaba primer año, un $34 \%$ segundo y el porcentaje restante asistía a tercer año. Un $67 \%$ señalaba que residía con ambos progenitores y el $78 \%$ de los adolescentes se percibía de clase socioeconómica media. Si bien la edad de consentimiento sexual en la Argentina es a partir de los 16 años, se decidió conservar a los adolescentes de 16 años debido a que eran un porcentaje menor ( 82 casos). Por otra parte, aunque legalmente ya tienen la edad de consentimiento, su inmadurez psicosocial frente a la de un adulto que los contacta con fines sexuales también los coloca en lugar de riesgo para una peor salud mental u otros tipos de riesgos, como la explotación sexual o la pornografía.

\section{Instrumentos}

Cuestionario sociodemográfico ad hoc. Género, edad, curso que asistía, entre otros.

Cuestionario de Solicitación e Interacción Sexual a menores online por parte de adultos (QOSSIA) de Gámez-Guadix et al. (2017). Este cuestionario de 10 ítems pide a los adolescentes que indiquen con qué frecuencia experimentaron una solicitud o interacción sexual en con un sujeto de 18 años o más durante el último año, utilizando una escala Likert de 4 puntos: 0 (nunca), 1 (una vez o dos veces), 2 (3-5 veces) y 3 (6 o más veces). Se inquiere sobre el año pasado para evitar el sesgo temporal debido a períodos más largos y porque dicho período de tiempo se ha utilizado en cuestionarios anteriores de temas semejantes (por ejemplo, Encuesta de seguridad en Internet para jóvenes de Jones et al., 2012), lo que favorece las comparaciones con anteriores resultados. Con respecto a sus propiedades psicométricas, los estudios en muestras de adolescentes españolas indicaron una adecuada estructura factorial con dos factores; uno denominado solicitaciones sexuales y el otro, interacciones sexuales. El primero estaba integrado por las primeras cinco preguntas que refieren a pedidos sexuales o solicitación por parte de un adulto hacia el adolescente (por ejemplo, el envío de una foto) y el otro se componía de las restantes, las cuales inquieren intenciones de parte del adulto para cometer un abuso sexual (por ejemplo, me encontré con un adulto que conocí en la internet). Dicha estructura explicaba un $60 \%$ de la varianza y fue confirmada en un análisis factorial confirmatorio que indicaba un buen ajuste, con TLI $=.98$, CFI $=.99$ y RMSEA $=.01$. La adaptación a la Argentina demostró buenas propiedades psicométricas en adolescentes, como adecuada estructura factorial similar a la de los autores, validez de constructo con respecto a los problemas emocionales, el sexting y la cybervictimización y confiabilidad por encima de .93 para la escala (Resett, 2019).

Cuestionario de Sexting (Gámez-Guadix, Almendros, Borrajo, \& Calvete, 2015). Este instrumento evalúa la frecuencia con la que los adolescentes enviaron contenidos sexuales online. Para diferenciar las conductas de sexting del envío de fotos e información como consecuencia del acoso (por ejemplo, tras recibir amenazas o insultos), se pide a los participantes que indiquen cuántas veces han hecho las siguientes cosas de manera voluntaria, es decir, porque ellos quieren o lo desean: 1) "Enviar información escrita o mensajes de texto con contenido sexual sobre ti"; 2) "Enviar fotos con contenido sexual (por ejemplo, desnudo/a) sobre ti"; y 3) "Enviar imágenes (por ejemplo, a través de webcam) o vídeos con contenido sexual sobre ti". Las opciones de respuesta son: $0=$ nunca $; 1=$ de 1 a 3 veces; $2=$ de 4 a 10 veces; y $3=$ más de 10 veces. La prevalencia se calcula considerando, al menos, aquellos participantes que marcan la opción de 1 a 3 veces o más. Es posible derivar un índice de sexting sumando o promediando las tres preguntas. Esta escala ha mostrado una adecuada validez de constructo con la personalidad y adecuada consistencia en muestras españolas y argentinas (Gámez-Guadix et al., 2015; Gámez-Guadix, De Santisteban, \& Resett, 2017). En la presente muestra el $\alpha$ de Cronbach fue .86.

Escala de Síntomas Psicosomáticos de Rosenberg (RPS, Rosenberg, 1965). Los 10 ítems de la RPS miden la ansiedad a partir de la activación del sistema nervioso autónomo sin incluir componentes cognitivos (Rosenberg, 1965). Consta de diez preguntas sobre la frecuencia con que se experimenta nerviosismo, insomnio, pesadillas, fuertes dolores de cabeza, temblor o transpiración de las manos, palpitaciones, problemas al respirar, aunque no se esté haciendo 
ejercicio, etcétera, que se puntúan de $0=$ nunca a $3=$ siempre. Un ejemplo de ítem es: "Me preocupan mis nervios". En la Argentina las $\alpha$ de Cronbach fluctúan de .74 a .78 (Facio, Resett, Mistrorigo, \& Micocci, 2006). En esta muestra el $\alpha$ de Cronbach fue .84.

El Inventario de Depresión de Kovacs para Niños y Adolescentes (CDI, Kovacs, 1992). El CDI es uno de los inventarios más usados para medir depresión en el mundo. Mide síndrome depresivo -a nivel de estado más que de rasgo- en niños y adolescentes de 7 a 17 años, a través de una serie de síntomas tales como estado de ánimo perturbado, anhedonia, disfunciones vegetativas, autoevaluación y conductas interpersonales distorsionadas. Consta de 27 ítems de tres alternativas cada uno que se puntúan de 0 a 2 en las cuales el sujeto debe escoger la que mejor se ajusta a cómo se ha sentido. Un ejemplo de ítems es: "Todo el tiempo me siento triste". En la Argentina, las $\alpha$ de Cronbach fluctúan de .86 a .89 (Facio et al., 2006). En la presente muestra el $\alpha$ de Cronbach fue .83 .

\section{Procedimiento}

El propósito de esta investigación fue explicado a los directivos de las escuelas y sus padres. Luego de obtener el consentimiento de la escuela y los padres -el de estos últimos por escrito-, se les informó a los participantes del propósito y se garantizó participación voluntaria, anonimato y confidencialidad. También se solicitó el consentimiento informado de los participantes. Los datos se recogieron en las horas de clases que las escuela destinaron para este fin y fue llevada a cabo por el equipo de investigación. Los participantes tardaron alrededor de 3040 minutos en completar los tests. La investigación fue aprobada por la Universidad Católica Argentina.

\section{Análisis de datos}

Los datos se analizaron con el programa Statistical Package for the Social Sciences (SPSS, IBM) versión 23 para sacar estadísticos descriptivos (porcentajes, medias, etc.) e inferenciales. Con respecto a los estadísticos inferenciales, se llevaron a cabo pruebas de $X^{2}$ y $t$ de student para comprobar si el género introducía diferencias en los puntajes de grooming y ANOVAs para determinar si la edad lo hacía. $X^{2}$ se usó para observar si el género introducía diferencias en cada una de las respuestas a las preguntas. El género y la edad se colocaron como factor entre sujetos y los puntajes de grooming como variable dependiente para la $t$ de student y el ANOVAs. Se llevaron a cabo unas comparaciones post hoc Games-Howell debido a que las varianzas eran heterogéneas $p s=.001$, como se sugiere (Howell, 2007). Se realizaron regresiones en bloque para observar si la edad y el género (primer bloque para controlar dichas variables) y los problemas emocionales y el sexting (segundo bloque) predecían las conductas de grooming. Estas variables se colocaron como predictores y los puntajes de grooming como variables dependientes. La muestra originalmente era de 752 casos, pero 25 participantes se eliminaron del presente análisis al ser outliers, ya que con respecto a la solicitudes o interacciones puntuaban $\mathrm{z}=+3.29(p<.001)$, como sugieren algunos autores (Tabachnick \& Fidell, 2014). Respecto a la distribución de las variables, para la ansiedad, depresión y sexting, la asimetría se hallaba en valores relativamente normales que iban de 0.07 a 2.50 , con respecto a la curtosis, estos iban de 0.88 a 5.20. Valores de asimetría mayores de 3 y de curtosis de 8 a 20 -o más- son extremos (Kline, 2015; Tabacknick \& Fidell, 2014). En cambio, para solicitudes los valores de asimetría y curtosis eran 3.01 y 8.36 , respectivamente, para las interacciones eran 4.32 y 12.60 , respectivamente, y para el grooming eran 3.32 y 12.30 , respectivamente, por ende, se apartaban de la normalidad. En ciencias sociales, como la psicología, no es atípico que se hallen distribuciones que se apartan de la normalidad, más aún en una variable como el grooming online. Por este motivo, se decidió llevar a cabo transformaciones logarítmicas debido a que existía una asimetría positiva hacia la izquierda con el fin de lograr una distribución más cercana a la normalidad (Howell, 2007). Aunque no todos los autores aceptan este procedimiento, las mismas pueden realizarse con este fin para poder llevar a cabo análisis 
estadísticos más precisos, principalmente cuando algunas variables tienen distribución normal, pero otras, no (Tabacknick \& Fidell, 2014), como en este caso. Además, la muestra era de un tamaño grande y las comparaciones de medias, como $t$ de student, son bastante robustas en este sentido (Sainani, 2012; Tabacknick \& Fidell, 2014). Luego de la transformación, la distribución mejoró de modo notable para las solicitudes, las interacciones y el grooming, con valores de asimetría y curtosis de 1.99 y 5.02 , de 2.13 y 6.28 y de 2.10 y 6.05 , respectivamente. Como los datos ahora se habían alejado de valores extremos, se decidió usar estadísticos paramétricos.

\section{Resultados}

En la Tabla 1 se presentan los porcentajes de respuestas para cada una de las preguntas según el género y para el total de los adolescentes que reportaron haber sufrido dicha conducta en, al menos, una ocasión. También se presentan los estadísticos descriptivos de solicitación, interacción y grooming totales. Como se muestra en la tabla, solamente emergían diferencias de género en la pregunta 8 y 10 debido a porcentajes más altos de los varones, en comparación con las mujeres $X^{2}(1)=9.86 p<.002$ y $X^{2}(1)=3.17 p<.05$, respectivamente.

Tabla 1

Porcentajes y estadísticos descriptivos de solicitación, interacción y grooming total en adolescentes

\begin{tabular}{|c|c|c|c|c|}
\hline Pregunta & Varones & Mujeres & Total & \\
\hline 1. Pedir fotos o videos de tipo sexual & $8 \%$ & $8 \%$ & $8 \%$ & \\
\hline $\begin{array}{l}\text { 2. Preguntas explicitas de contenido sexual } \\
\text { desde internet o el celular }\end{array}$ & $9 \%$ & $9 \%$ & $9 \%$ & \\
\hline 3. Pedir cibersexo (por ej., con webcam) & $6 \%$ & $4 \%$ & $5 \%$ & \\
\hline $\begin{array}{l}\text { 4. Pedir encontrarnos en persona para tener } \\
\text { sexo }\end{array}$ & $6 \%$ & $4 \%$ & $5 \%$ & \\
\hline 5. Me enviaron fotos o videos de tipo sexual & $12 \%$ & $10 \%$ & $11 \%$ & \\
\hline 6. Envié fotos o videos míos de tipo sexual & $4 \%$ & $3 \%$ & $3 \%$ & \\
\hline $\begin{array}{l}\text { 7. He coqueteado o mantenido un contacto } \\
\text { romántico con un adulto online }\end{array}$ & $6 \%$ & $3 \%$ & $4 \%$ & \\
\hline $\begin{array}{l}\text { 8. He hablado de cosas sexuales con un adulto } \\
\text { en internet o el celular }\end{array}$ & $14 \%$ & $4 \%$ & $8 \%$ & \\
\hline $\begin{array}{l}\text { 9. Me encontré con un adulto que conocí } \\
\text { online }\end{array}$ & $7 \%$ & $4 \%$ & $5 \%$ & \\
\hline $\begin{array}{l}\text { 10. Me encontré con un adulto para tener } \\
\text { contacto sexual }\end{array}$ & $5 \%$ & $2 \%$ & $3 \%$ & \\
\hline Dimensión & Mín & Máx & $M$ & $D E$ \\
\hline Solicitación & 0 & 15 & 0.54 & 1.84 \\
\hline Interacción & 0 & 15 & 0.43 & 1.80 \\
\hline $\begin{array}{l}\text { Grooming } \\
N=727\end{array}$ & 0 & 30 & 0.98 & 3.46 \\
\hline
\end{tabular}

Con respecto al porcentaje total, un $17 \%$ de los adolescentes informó sufrir alguna experiencia de solicitación, un 12\%, de interacción y un 20\%, de grooming (ambos). No existían diferencias de género en dichas variables, con la sola excepción de interacción en la cual emergían diferencias marginales debido a porcentajes más alto de los varones en comparación con las mujeres $14 \%$ versus $10 \% X^{2}(1)=2.94 p=.09$.

En la Tabla 2, se presentan las medias y desvíos típicos en solicitación, interacción y puntajes totales de grooming, según género. 
Tabla 2

Medias y desvios típicos en solicitación, interacción y grooming total según género

\begin{tabular}{lllll}
\hline \multirow{3}{*}{ Solicitación } & Género & $N$ & $M$ & $D E$ \\
& varón & 282 & 0.68 & 2.33 \\
& mujer & 445 & 0.47 & 1.48 \\
Interacción & varón & 282 & 0.68 & 2.26 \\
& mujer & 445 & 0.28 & 1.45 \\
Grooming & varón & 282 & 1.36 & 4.44 \\
& mujer & 445 & 0.75 & 2.70 \\
\hline
\end{tabular}

Al comparar los puntajes con $t$ de student para las tres variables según el género, las varianzas de ambos grupos eran homogéneas $p \mathrm{~s}>.13 \mathrm{y}$ se hallaban diferencias en interacción y marginales en grooming $t(725)=2.30 p<.05$ y $t(725)=1.62 p=.09$, respectivamente, debido a mayores niveles de los varones que lo padecían, como se presenta en la Tabla 2.

En la Tabla 3 se presentan las medias y desvíos estándar en solicitación, interacción y grooming según grupo de edad agrupándolos en tres grupos de edades equivalentes: 11-12, 13-14 y 15-16 años. Al realizar un ANOVA con la edad como factor entre sujetos y los puntajes de grooming como variables dependientes, emergían diferencias significativas. Al realizar comparaciones post hoc, las diferencias se debían a que el grupo de edad 11-12 y 13-14 presentaba menores niveles de solicitación, interacciones y grooming, en comparación con el grupo de 15-16 años $p \mathrm{~s}<.004, .05, .001, .05, .001$ y .04, respectivamente. Se hallaba una función lineal entre la edad y los puntajes de solicitación, interacciones y grooming $\mathrm{F}=10.59 p<001 ; \mathrm{F}=17.62 p<$ .001 y F $=15.41 p<.001$, respectivamente, como se muestra en la Figura 1.

Tabla 3

Medias y desvíos típicos en solicitación, interacción y grooming total según grupo de edad

\begin{tabular}{llllll}
\hline & & $N$ & $M$ & $D E$ & \\
\hline Grooming & Edad 11-12 & 292 & 0.50 & 1.89 & $\mathrm{~F}=8.02 * * *$ \\
& Edad 13-14 & 223 & 0.90 & 3.42 & \\
& Edad 15-16 & 212 & 1.73 & 4.80 & \\
& Total & 727 & 0.98 & 3.46 & \\
\hline Solicitación & Edad 11-12 & 292 & 0.34 & 1.35 & $\mathrm{~F}=5.84^{* *}$ \\
& Edad 13-14 & 223 & 0.47 & 1.64 & \\
& Edad 15-16 & 212 & 0.90 & 2.48 & \\
& Total & 727 & 0.54 & 1.84 & \\
\hline Interacción & Edad 11-12 & 292 & 0.15 & .67 & $\mathrm{~F}=8.90^{* * *}$ \\
& Edad 13-14 & 223 & 0.43 & 1.92 & \\
& Edad 15-16 & 212 & 0.83 & 2.53 & \\
& Total & 727 & 0.43 & 1.80 & \\
\hline
\end{tabular}

Nota. $* * * p<.001 * * p<.003$ 


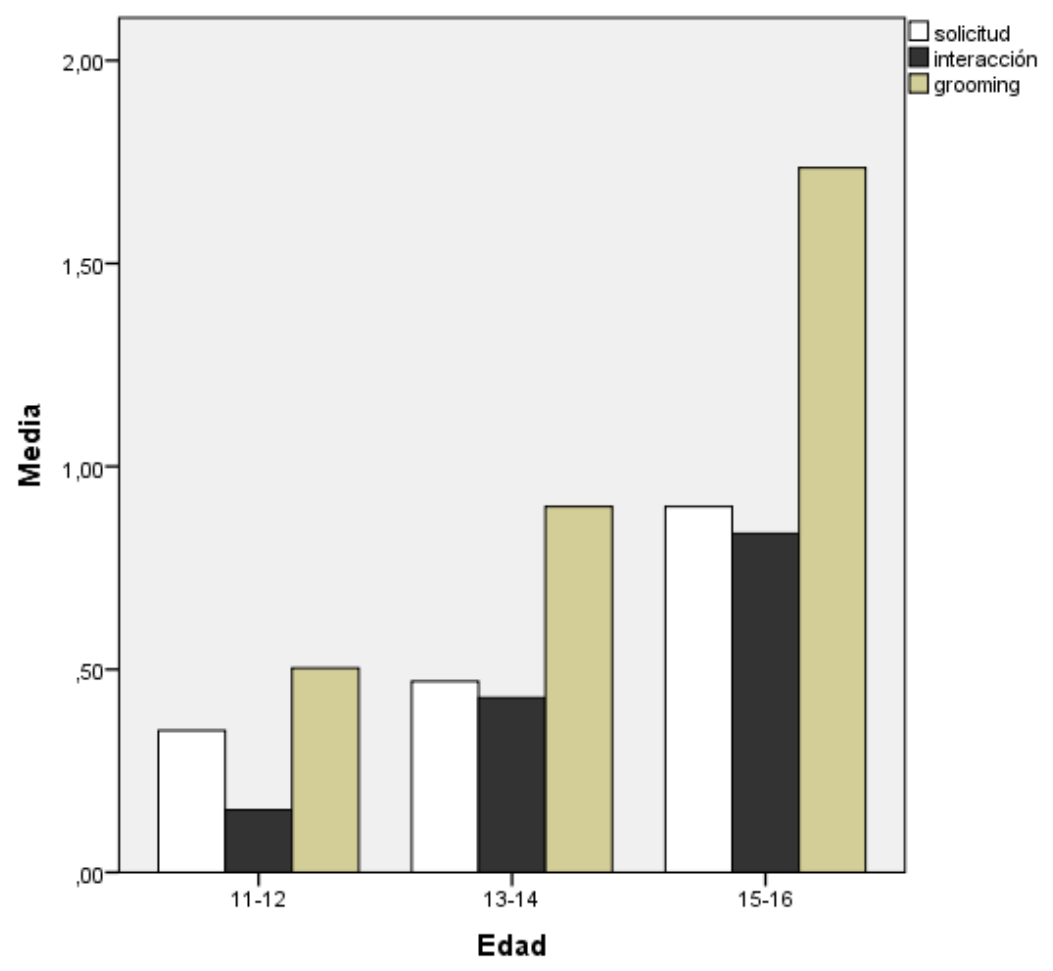

Figura 1. Medias en solicitación, interacción y grooming total en adolescentes según edad.

Se llevaron a cabo una serie de regresiones en bloques con las edades (12 años a 16 años) y el género $(0=$ mujer y $1=$ varón $)$ en el primer bloque, y los puntajes en depresión, ansiedad y sexting en el segundo con el fin de predecir los puntajes de solicitación, interacción y grooming. En la Tabla 4 se presentan los resultados. Tanto para la solicitación, como para la interacción y el grooming, ambos bloques eran significativos $p \mathrm{~s}<.005$. Como se ve en dicha tabla, se predecía un $19 \%$ de la varianza en solicitación, un $22 \%$ en interacción y un $23 \%$ en grooming, con los predictores significativos siendo la edad, depresión y sexting. El primer bloque predecía 5\%, 4\% y $6 \%$, por lo cual se generaba un incremento significativo en la predicción. El género era marginalmente significativo para la solicitación. Como se muestra en la Tabla 4, a mayor edad, mayores niveles de grooming, a mayores puntajes de depresión, mayores niveles de grooming y a mayor sexting, mayores puntajes de dicha conducta. Marginalmente el ser varón predecía mayor nivel de solicitación. 
Tabla 4

Resultados de las regresiones en bloque para predecir la solicitación, interacción y grooming a partir de género, edad, problemas emocionales y sexting en adolescentes

\begin{tabular}{|c|c|c|c|c|c|}
\hline & $\begin{array}{l}\text { Primer } \\
\text { bloque } \\
\text { Género }\end{array}$ & Edad & $\begin{array}{l}\text { Segundo } \\
\text { bloque } \\
\text { Depresión }\end{array}$ & Ansiedad & Sexting \\
\hline Solicitación & $\beta=.21$ & $\beta=.12$ & $\beta=.88$ & $\beta=.18$ & $\beta=.34$ \\
\hline$R^{2}=19 \%$ & $t=1.65^{*}$ & $t=2.39 * *$ & $t=3.99 * * *$ & $t=1.18$ & $t=6.92^{* * *}$ \\
\hline $\begin{array}{c}\text { Intervalos de } \\
\text { confianza al } \\
95 \%\end{array}$ & $-.70 / .05$ & $.01 / .22$ & $.02 / .11$ & $-.01 / .03$ & $.36 / .66$ \\
\hline $\begin{array}{c}\text { Interacción } \\
R^{2}=22 \%\end{array}$ & $\begin{array}{c}\beta=-.01 \\
t=-.01\end{array}$ & $\begin{array}{c}\beta=.15 \\
t=3.20^{* * * *}\end{array}$ & $\begin{array}{c}\beta=.66 \\
t=3.08 * * *\end{array}$ & $\begin{array}{c}\beta=-.02 \\
t=-.13\end{array}$ & $\begin{array}{c}\beta=.38 \\
t=8.06 * * *\end{array}$ \\
\hline $\begin{array}{c}\text { Intervalos de } \\
\text { confianza al } \\
95 \%\end{array}$ & $-.82 /-.10$ & $.06 / .25$ & $.01 / .08$ & $-.02 / .03$ & $.44 / .72$ \\
\hline $\begin{array}{c}\text { Grooming } \\
R^{2}=23 \%\end{array}$ & $\begin{array}{c}\beta=.10 \\
t=.88\end{array}$ & $\begin{array}{c}\beta=.14 \\
t=2.98 * * *\end{array}$ & $\begin{array}{c}\beta=.81 \\
t=3.79 * * *\end{array}$ & $\begin{aligned} \beta & =.08 \\
t & =.58\end{aligned}$ & $\begin{array}{c}\beta=.38 \\
t=7.98 * * *\end{array}$ \\
\hline $\begin{array}{c}\text { Intervalos de } \\
\text { confianza al } \\
95 \%\end{array}$ & $-1.48 /-.10$ & $.08 / .45$ & $.02 / .19$ & $-.03 / .05$ & $.82 / 1.36$ \\
\hline
\end{tabular}

\section{Discusión}

La importancia de este trabajo era examinar el nivel de grooming online, con sus aspectos de solicitaciones e interacciones, en una muestra de adolescentes argentinos y determinar si el género, la edad, los problemas emocionales -depresión y ansiedad- y el sexting eran predictores del mismo. Para este fin, se constituyó una muestra intencional de 727 adolescentes entre 12 y 16 años (59\% mujeres y $41 \%$ varones) que cursaban de primer año a tercer año de dos escuelas privadas de la ciudad de Paraná, Argentina. Los participantes contestaron un cuestionario de grooming online, medidas de problemas emocionales y un cuestionario de sexting.

Se detectó que un $17 \%$ de los adolescentes informaban solicitaciones, un $12 \%$, interacciones y un $20 \%$, casos de grooming online al menos una vez en el último año. Dichos porcentajes eran más altos que los de $13 \%$ y $8 \%$ de solicitación e interacción sexual hallados con el mismo instrumento en muestras españolas por el autor del test (Gámez-Guadix et al., 2017). También eran más elevados a los de 5\%-15\% hallados en adolescentes de 10 a 17 años en los Estados Unidos y Europa (Bergen et al., 2014). Sin embargo, los datos aquí detectados revisten de una gran preocupación debido a que los porcentajes eran más altos que detectados en otros estudios internacionales. Por otra parte, los niveles pueden ser mucho más elevados, ya que muchos adolescentes pueden no haber confesado el grooming online para dar respuestas socialmente deseables, por miedo o vergüenza, entre otros motivos (Gámez-Guadix, 2017). Por ejemplo, muchos adolescentes pueden no haberlo señalado en el cuestionario por miedo a ser reprendidos o-incluso- porque los mismos adultos con lo que tuvieron contacto los amenazaron o les dijeron que eso era un "secreto" entre ellos, la cual es una de sus estrategias de persuasión (De Santiesteban \& Gámez-Guadix, 2017; Gámez-Guadix, Almendros, Calvete, \& De Santisteban, 2018). Del mismo modo, hay que tener en cuenta que los adultos también pueden haber mentido en su edad - por ejemplo, decir que eran menores- y, así, muchos más participantes pueden haberlo sufrido sin percatarse de ello.

Diversos factores culturales, sociales y económicos podrían explicar las diferencias halladas entre los porcentajes de grooming aquí informados y los del autor del test en España. Por 
ejemplo, la Argentina es un país en el cual los adolescentes tienen una gran fascinación por las nuevas tecnologías, como los celulares, y -a nivel de la América Latina- se encuentran entre quienes más usan dichas tecnologías; por este motivo, han sido llamados la generación interactiva (Facio \& Resett, 2012; Fundación Telefónica, 2008). A muchos adolescentes argentinos, inclusive, son sus mismos progenitores quienes les compraron el primer celular cuando eran niños. Por ejemplo, en la Argentina los niños ya tienen celular antes de los 9 años de edad y es el país de la región donde más tempranamente ocurre este hecho, como sugiere un reciente estudios del Fondo para la Infancia de las Naciones Unidas (UNICEF, 2020). Incluso dicho estudio indicaba que los adolescentes señalaban que sus padres y docentes hacían muy poco para protegerlos de los peligros de la Internet. Es posible que, de este modo, también diferencias culturales en la educación parental expliquen estos resultados. No obstante, al tratarse aquí de una muestra intencional los hallazgos deben ser tomados con cautela.

Con respecto a las diferencias de género, se hallaron diferencias de medias en interacción y marginales en grooming debido a que más varones lo sufrían -lo mismo se detectaba al comparar los porcentajes de interacciones, ya que $14 \%$ de varones lo sufrían versus $10 \%$ de las mujeres-. Si bien las diferencias de género en algunos estudios señalan que las mujeres lo sufren en mayor medida (Mitchell et al., 2008; Wolak et al., 2010), las investigaciones con el presente instrumento en España también detectaron que eran los varones quienes lo sufrían en mayor medida. Una posible explicación de dichas diferencias-como en la pregunta me encontré con un adulto en persona para tener sexo, la cual es una de las que mide interacciones- puede deberse a que ellos son más desinhibidos y tienen menor temor de ser agredidos sexualmente en comparación con las mujeres.

El tema de las diferencias de género debe ser examinado en mayor detalle, pero es posible que la inconsistencia en los hallazgos pueda deberse a las distintas muestras y operacionalizaciones del constructo, principalmente en un tema como en el grooming online en que las investigaciones son todavía incipientes. Por otra parte, también es posible que las mujeres lo informen en menor medida por una mayor deseabilidad social o por temor a ser desaprobadas socialmente, principalmente en una nación latina como la Argentina en la cual la doble moral con respecto a la sexualidad está muy presente, con muchos autores indicando que esto sucede con frecuencia en sociedades más tradicionales (Ybarra \& Mitchell, 2014). Del mismo modo, está establecido que las mujeres adolescentes presentan una mayor deseabilidad social en comparación con los varones (Facio et al., 2006).

Con respecto a la edad, se detectó una función lineal entre la edad y solicitación, interacción y grooming debido a que mayor edad, mayor padecimiento de dichas conductas, con los adolescentes de 15-16 años informando los niveles más altos. Dichos estudios son coincidentes con los de otros estudios internacionales que encontraron que, a mayor edad, mayor nivel de grooming (Gámez-Guadix et al., 2017). Está comprobado que los pedófilos buscan víctimas menores, pero de una edad mayor que la de los niños de edad escolar porque ellos hacen un mayor uso de las TIC con fines de comunicación y con menor supervisión adulta, en comparación con los niños (Gamez-Guadix, 2017). Que la edad de los adolescentes se asocie linealmente con el grooming puede deberse a que a través de la edad crece linealmente el uso de las TIC por parte de ellos. Por ejemplo, en Europa un 38\% de los menores de 9-12 eran usuarios de redes sociales versus el 77\% de los de 13-16 (Livingstone, Olafsson, \& Staksrud, 2011). Por otra parte, también los agresores, muchas veces, prefieren víctimas que hayan atravesados los cambios puberales debido a que el cuerpo adolescente cuenta ya con las proporciones adultas. Del mismo modo, los cambios sociales y puberales que los adolescentes van experimentando a medida que entran en esta etapa llevan a los adolescentes a mostrar un mayor interés y contacto sexual (Steinberg, 2010). También el incremento con la edad puede deberse a que los adolescentes -a medida que ingresan en esta importante etapa de la vida- no solamente incrementan el uso de TIC para fines de comunicación e intercambios sociales (Gámez-Guadix, 2017), sino también para explorar su 
sexualidad e identidad (Arnett, 2014). Se sabe que el uso de las TIC puede ser un modo frecuente para los adolescentes de explorar su sexualidad a medida que se desarrollan, como señalan algunos autores (Cooper, Quayle, Jonsson, \& Svedin, 2016; Döring, 2014; Walker, Sanci, \& TempleSmith, 2013).

Con respecto a la predicción de dicha conducta, se halló una varianza explicada de tamaño grande -según los tamaños de efectos de Cohen (1992)- con 19\% 22\% y 23\%, para solicitud, interacción y grooming, respectivamente, a partir del género, la edad y los problemas emocionales y el sexting. Nuevamente el ser varón y la mayor edad se asociaban significativamente con sufrir dicha conducta, aunque para el primero la significación era marginal. No se hallaban asociaciones entre dicha conducta y la ansiedad, pero sí para la depresión; lo cual indicaría que adolescentes con una sintomatología depresiva -una imagen negativa de sí mismo, por ejemplo- serían sujetos más vulnerables al grooming online, como detectaron algunas investigaciones (por ejemplo, Montiel et al., 2016; Ybarra et al., 2004). Futuros estudios deberían examinar si la ansiedad excesivo temor o preocupación- es un factor de riesgo o no para el grooming. Por ejemplo, quizás altos niveles de ansiedad -con su componente de preocupación excesiva- se asocien con no hablar con extraños en la Internet. Lo importante de estos hallazgos es que la sintomatología depresiva era un predictor significativo luego de controlar el género, ya que está comprobado que varones y mujeres difieren con respecto a la sintomatología depresiva (Facio et al., 2006; Steinberg, 2010).

La alta asociación entre el grooming y el sexting no es llamativa -era el mejor predictor junto con la edad-, ya que el compartir contenidos sexuales personales podría ser un factor de riesgo para que los adultos contacten a los menores con fines sexuales. Estudios recientes (Machimbarrena et al., 2018; Reyns et al., 2013) también detectaron dicha asociación, como también la asociación entre el grooming online y la cybervictimización -otra forma de victimización, pero a mano de los pares-. Está establecido que los adultos que llevan a cabo el grooming buscan información sobre la vulnerabilidad de las posibles víctimas antes de iniciar el abuso (Gámez-Guadix et al., 2018), por ejemplo, mediante el estudio sobre el contenido que los adolescentes exponen en sus redes sociales. De este modo, el compartir contenidos íntimos, como fotos, videos o la sintomatología depresiva -tristeza extrema, estar disconforme con uno mismo, entre otros indicadores- puede ser un antecedente del grooming. Aunque también es posible que dichas conductas sean también una consecuencia del grooming, esto es, el inadecuado contacto sexual con un adulto podría volver a los menores más desinhibidos en lo sexual -como ocurre con el abuso sexual físico- e incrementar sus conductas de sexting (Paolucci, Genuis, \& Violato, 2001). Futuros estudios deberían examinar las relaciones temporales entre dichas conductas con el fin de examinar a través del tiempo la estabilidad del fenómeno como de la direccionalidad de las variables, lo cual no es posible en un tipo de estudio transversal. Estudios recientes longitudinales españoles demostraron relaciones bidireccionales entre el grooming online y el sexting (GámezGuadix \& Mateo-Pérez, 2019), aunque más investigación es necesaria con el fin de detectar cómo es el mecanismo entre dichas variables. Sería necesario que futuras investigaciones indaguen sobre cómo es la direccionalidad de las variables de forma más precisa y en una nación con gran uso de las TIC en adolescentes. Asimismo, podría existir bireccionalidad entre las variables, pero, en este caso, ¿existe una direccionalidad más significativa? $\mathrm{O}$ en otras palabras y usando -por ejemploecuaciones estructurales, ¿cuál de los senderos es más significativo? Esto es, ¿es el sexting un antecedente o una consecuencia del grooming online? Este tipo de estudio es crucial para las actividades de prevención.

El presente trabajo tiene una serie de limitaciones que deben ser destacadas. En primer lugar, una selección de la muestra intencional de Paraná, Entre Ríos, Argentina; el uso del autoinforme para medir el grooming online -el cual tiene limitaciones conocidas, principalmente en una variable en la cual la falta de honestidad o deseabilidad social tienen una gran repercusióny con una muestra de un tamaño menor a la empleada en España por los autores del test. También el haber evaluado todas las variables con el autoinforme aumenta artificialmente las correlaciones 
entre las variables por la varianza compartida por el método de recolección de datos. Por otra parte, el sexting se midió solamente con una escala de tres preguntas. Finalmente, el tipo de estudio transversal no permite determinar la direccionalidad de la causalidad.

Futuros estudios deberían examinar esta problemática en muestras de mayor tamaño y seleccionadas al azar de la Argentina, con el fin de poder generalizar los resultados. Del mismo modo, sería interesante realizar estudios en otros países de la región para comparar los resultados y determinar si la Argentina presenta los niveles más altos de la región o no. También sería interesante incluir otras edades, como niños de edad escolar, para saber si a edades tempranas como los 10 u 11 años- ya existen víctimas de grooming, por ejemplo, está estudiado que en la Argentina ya a los 9 años los niños tienen teléfonos celulares. Futuras investigaciones deberían ser longitudinales para determinar la estabilidad temporal del grooming online, las solicitudes y las interacciones, como evaluar las interrelaciones entre dicha conducta y el sexting, por ejemplo, ¿el grooming es un antecedente o una consecuencia del sexting? Asimismo, se debería examinar dicha conducta con otras técnicas de recolección de datos, como las nominaciones de pares para evitar las limitaciones del autoinforme, principalmente ante conductas negativas como el sexting o el grooming. Por otra parte, futuras investigaciones deberían examinar las relaciones entre las variables con modelos estructurales. Sería deseable evaluar la influencia de otras variables de relevancia, como la sexualidad precoz, uso problemático de nuevas tecnologías, phubbing, rasgos de personalidad, impulsividad, como factores interpersonales - presión del grupo de pares, problemas familiares, etc.-, entre otras. También sería interesante ver cuáles dimensiones parentales se asocian con tener hijos adolescentes que sufren de dicha conducta -estilos de crianza, control parental, falta de supervisión parental de las TIC, entre otras- o si el uso problemático de TIC por parte de los padres -uso excesivo por parte de ellos, por ejemplo- se relacionan con el grooming padecido. Sería interesante ver cómo las dimensiones de la parentalidad conllevarían a sufrir grooming, esto es, ¿hay un efecto directo o es un efecto indirecto a través del uso inadecuado de las nuevas tecnologías? Principalmente, observando que los niveles de grooming aquí detectados son más elevados que los hallados en España. Finalmente, se debería avanzar en la prevención de la problemática. Estos resultados indicarían que factores como sintomatología depresiva, mayor edad y el compartir contenidos íntimos de contenido sexual pueden asociarse con el grooming. Por lo cual, estas conductas deben ser observadas con cuidado por parte de los padres, docentes y los adultos. No con un mensaje de prohibir el uso de las TIC por parte de los adolescentes, lo cual sería poco realista (Gámez-Guadix, 2017), sino con la idea de un uso seguro de las mismas y tomando precauciones. También se debería avanzar en el desarrollo de programas o juegos online -de gran atracción para los adolescentes- que concienticen en el uso de seguro de TIC y los riesgos de un mal empleo de estas, como puede ser el grooming y el sexting.

\section{Referencias}

Agustina, J. R., \& Gómez-Durán, E. L. (2012). Sexting: Research criteria of a globalized social phenomenon. Archives of Sexual Behavior, 41, 1325-1328. doi: 10.1007/s10508-0120038-0

Arnett, J. J. (2014). Adolescence and Emerging Adulthood. A cultural approach. Nueva Jersey: Prentice Hall.

Barrense-Dias, Y., Berchtold, A., Surís, J., \& Akre, C. (2018). Sexting and the Definition Issue. Journal of Adolescent Health, 61(5), 544-554.

Baumgartner, S., E., Valkenburg, P.M., \& Peter, J. (2010). Unwanted online solicitation and risky sexual online behavior across the lifespan. Journal of Applied Developmental Psychology, $31,439-447$. 
Benotsch, E., Snipes, D., Martin, A., \& Bull, S. (2012). Sexting, Substance Use, and Sexual Risk Behavior in Young Adults. The Journal of adolescent health: official publication of the Society for Adolescent Medicine, 52(3), 307-313, doi: 10.1016/j.jadohealth.2012.06.011.

Bergen, E., Davidson, J., Schulz, A., Schuhmann, P., Johansson, A., Santtila, P., \& Jern, P. (2014). The effects of using identity deception and suggesting secrecy on the outcomes of adultadult and adult-child or-adolescent online sexual interactions. Victims \& Offenders, 9(3), 276-298. doi: 10.1080/15564886.2013.87375

Cohen, J. (1992). Quantitative methods in psychology: A power primer. Psychological Bulletin, 112, 155-159.

Cooper, K., Quayle, E., Jonsson, L., \& Svedin, C. G. (2016). Adolescents and self-taken sexual images: A review of the literature. Computers in Human Behavior, 55, 706-716.

Crimmins, D. M., \& Siegfried-Spellar, K.C. (2014). Peer attachment, sexual experiences, and risky online behaviors as predictors of sexting behaviors among undergraduate students. Computers in Human Behavior, 32, 268-275.

De Santisteban, P., \& Gámez-Guadix, M. (2017). Estrategias de persuasión en grooming online de menores: un análisis cualitativo con agresores en prisión. Psychosocial Intervention, 26, 139-146. doi: 10.1016/j.psi.2017.02.001

Döring, N. (2014). Consensual sexting among adolescents: Risk prevention through abstinence education or safer sexting. Cyberpsychology, 8(1), 1-18. doi: 10.5817/CP2014-1-9

Facio, A., \& Resett, S. (2012). Argentina. En J. Arnett (Ed.), Adolescent Psychology around the World, (pp. 151-162). Nueva York: Psychological Press.

Facio, A., Resett, S., Mistrorigo, C., \& Micocci, F. (2006). Los adolescentes argentinos. Cómo piensan y sienten. Buenos Aires: Editorial Lugar.

Fondo de las Naciones Unidas para la Infancia UNICEF (2020). Los y las adolescentes. Su percepción sobre la seguridad online. Relevamiento 2020. Buenos Aires: UNICEF.

Fundación Telefónica (2008). La generación interactiva en Iberoamérica. Barcelona: Ariel.

Gámez-Guadix, M. (2017). Escuela de padres 3.0. Guía para educar a los niños en el uso positivo de Internet. Madrid: Pirámide.

Gámez-Guadix, M., Almendros, C., Borrajo, E., \& Calvete, E. (2015). Prevalence and Association of Sexting and Online Sexual Victimization Among Spanish Adults. Sexuality Research and Social Policy, 12(2), 145-154.

Gámez-Guadix, M., Almendros, C., Calvete, E., \& De Santisteban, P. (2018). Persuasion strategies and sexual solicitations and interactions in online sexual grooming of adolescents: Modeling direct and indirect pathways. Journal of Adolescence, 63, 11-18. doi: 10.1016/j.adolescence.2017.12.002

Gámez-Guadix, M., De Santisteban, P., \& Alcazar, M. (2017). The construction and psychometric properties of the questionnaire for online sexual solicitation and interaction of minors with adults. Sexual Abuse: A Journal of Research and Treatment, 30(8), 1-17. doi: $10.1177 / 1079063217724766$

Gámez-Guadix, M., De Santisteban, P., \& Resett, S. (2017). Sexting among Spanish adolescents: Prevalence and personality profiles. Psicothema, 29(1), 29-34. doi: 10.7334/psicothema2016.222

Gámez-Guadix, M., \& Mateo-Pérez, E. (2019). Longitudinal and reciprocal relationships between sexting,online sexual solicitations, and cyberbullying among minors. Computers in Human Behavior, 94, 70-76.

Howell D. (2007). Statistical method for psychology. Belmont, CA: Thomson Wadsworth.

Jones, L. M., Mitchell, K. J., \& Finkelhor, D. (2012). Trends in youth Internet victimization: Findings from three youth Internet safety surveys 2000-2010. Journal of Adolescent Health, 50, 179-186. doi: 10.1016/j.jadohealth.2011.09.015

Kline, R. (2015). Principles and practice of structural equation modeling. Nueva York: Guilford. 
Kloess, J. A., Beech, A. R., \& Harkins, L. (2014). Online child sexual exploitation prevalence, process, and offender characteristics. Trauma, Violence, \& Abuse, 15, 126-139. doi: $10.1177 / 1524838013511543$

Kovacs, M. (1992). Children's Depression Inventory Manual. North Tonawanda: Multi-Health Systems.

Livingstone, S., Olafsson, K., \& Staksrud, E. (2011). EU Kids online: Social networking, age and privacy. Recuperado de: www.eukidsonline.net.

Machimbarrena, J., Calvete, E., Fernández-González, L., Álvarez-Bardón, A., Álvarez-Fernández, L. \& González-Cabrera, J. (2018). Internet Risks: An Overview of Victimization in Cyberbullying, Cyber Dating Abuse, Sexting, Online Grooming and Problematic Internet Use. International Journal of Environmental Research and Public Health, 15, 1-15

Manlove, J., Terry-Humen, E., \& Ikramullah, E. (2006). Young teenagers and older sexual partners: Correlates and consequences for males and females. Perspectives on Sexual and Reproductive Health, 38, 197-207. doi: 10.1363/psrh.38.197.06

McRae, K., Gross, J. J., Weber, J., Robertson, E. R., Sokol-Hessner, P., Ray, R. D., Gabrieli, J. D. E., \& Ochsner, K. N. (2012). The development of emotion regulation: An fMRI study of cognitive reappraisal in children, adolescents and young adults. Social Cognitive and Affective Neuroscience, 7, 11-22. doi: 10.1093/scan/nsr093

Mitchell, K. J., Finkelhor, D., Jones, L. M., \& Wolak, J. (2012). Prevalence and characteristics of youth sexting: a national study. Pediatrics, 129(1), 13-20.

Mitchell, K. J., Finkelhor, D., \& Wolak, J. (2007). Youth Internet users at risk for the most serious online sexual solicitations. American Journal of Preventive Medicine, 32, 532-537. doi: 10.1016/j.amepre.2007.02.001

Mitchell, K. J., Wolak, J., \& Finkelhor, D. (2008). Are blogs putting youth at risk for online sexual solicitation or harassment? Child Abuse \& Neglect, 32, 277-294. doi: 10.1016/j. chiabu.2007.04.015

Montiel, I., Carbonell, E., \& Pereda, N. (2016). Multiple online victimization of Spanish adolescents: Results from a community sample. Child Abuse \& Neglect, 52, 123-134.

Morelli, M., Bianchi, D., Baiocco, R., Pezzuti, L., \& Chirumbolo, A. (2016). Sexting, psychological distress and dating violence among adolescents and young adults. Psicothema, 28(2), 137-142. doi: 10.7334/psicothema2015.193

Paolucci, E.O., Genuis, M.L., \& Violato,C. (2001). A meta-analysis of the published research on the effects of child sexual abuse. Journal of Psychology, 135, 17-36. doi: 10.1080/00223980109603677.

Quesada, S., Fernández-González, L., \& Calvete, E. (2018) Sexting en la Adolescencia: Prevalencia y Asociación con la Victimización de Acoso Online y Violencia en el Noviazgo. Psicología Conductual, 26(2), 225-242.

Resett, S. (2019). Análisis psicométrico del Cuestionario de Solicitación e Interacciones Sexuales en Menores. Paper en evaluación.

Reyns, B.W., Burek, M.W., Henson, B., \& Fisher, B.S. (2013). The unintended consequences of digital technology: Exploring the relationship between sexting and cybervictimization. Journal of Crime and Justice, 36(1), 1-17. doi: 10.1080/ 0735648X.2011.641816.

Ricketts, M., Maloney, C., Marcum, C., \& Higgins, G. (2015). The Effect of Internet Related Problems on the Sexting Behaviors of Juveniles. American Journal of Criminal Justice, 40(2), 270-284.

Rosenberg, M. (1965). Society and the adolescent self-image. Princeton, NJ: Princeton University Press.

Sainani, K. (2012). Dealing with non-normal data. $P M \& R \quad 4(12)$, 1001-1005. doi: 10.1016/j.pmrj.2012.10.013 
Smith, P. K., Thompson, F., \& Davidson, J. (2014). Cyber safety for adolescent girls: Bullying, harassment, sexting, pornography, and solicitation. Current Opinion in Obstetrics and Gynecology, 26, 360-365.

Steinberg, L. (2010). Adolescence. Nueva York: McGraw Hill.

Tabachnick, B. G., \& Fidell, L. S. (2014). Using multivariate statistics. Massachusetts: Allyn \& Bacon/Pearson Education.

Wachs, S., Whittle, H.C., Hamilton-Giachritsis, C., Wolf, K.D., Vazsonyi, A.T., \& Junger, M. (2017). Correlates of mono-and dual-victims of cybergrooming and cyberbullying: Evidence from four countries. Cyberpsychology, Behavior, and Social Networking, 21(2), 91-98. doi: 10.1089/cyber.2016.0733.

Walker, S., Sanci, L., \&Temple-Smith, M. (2013). Sexting: Young women's and men's views on its nature and origins. Journal of Adolescent Health, 52(6), 697-701. doi: 10.1016/j.jadohealth.2013.01.026.

Wolak, J., Finkelhor, D., Mitchell, K. J., \& Ybarra, M. L. (2010). Online "predators" and their victims: Myths, realities, and implications for prevention and treatment. Psychology of Violence, 1, 13-35. doi: 10.1037/2152-0828.1.S.13

Wurtele, S. \& Kenny, M. (2016). Technology-Related Sexual Solicitation of Adolescents: A Review of Prevention Efforts. Child Abuse Review, 25, 332-344. doi: 10.1002/car.2445

Ybarra, M.L., Leaf, P.J., \& Diener-West, M. (2004). Sex Differences in Youth-Reported Depressive Symptomatology and Unwanted Internet Sexual Solicitation. Journal of Medical Internet Research, 6(1), 5. doi: 10.2196/jmir.6.1.e5

Ybarra, M. L., \& Mitchell, K.J. (2008). How risky are social networking sites? A comparison of places online where youth sexual solicitation and harassment occur us. Pediatrics, 121(2), 350-357.

Contribución de los autores: a) Concepción y diseño del trabajo; b) Adquisición de datos; c) Análisis e interpretación de datos; d) Redacción del manuscrito; e) revisión crítica del manuscrito. S. R. ha contribuido en $a, b, c, d$, e.

Editora científica responsable: Dra. Cecilia Cracco 